\title{
Efficacy of Granulocyte Colony Stimulating Factor in Severe Alcoholic Hepatitis: A Systematic Review and Meta-Analysis
}

\author{
Muhammad Baig $^{1}$, Saqib Walayat ${ }^{1}$, Sonu Dhillon ${ }^{1}$, Srinivas Puli ${ }^{1}$ \\ 1. Gastroenterology, OSF St. Francis Medical Center/University of Illinois College of Medicine at Peoria, Peoria, USA
}

Corresponding author: Muhammad Baig, muhammad.baig@osfhealthcare.org

\section{Abstract \\ Background}

Severe alcoholic hepatitis is a condition with a very high mortality rate and there is a paucity of evidence regarding efficacy and safety of most available therapeutic options. The present systematic review and meta-analysis aims to assess the survival benefit of granulocyte colony stimulating factor (G-CSF) in patients with severe alcoholic hepatitis.

\section{Methods}

Studies involving adult patients receiving G-CSF for severe alcoholic hepatitis were searched in MEDLINE, Ovid journals, MEDLINE nonindexed citations, and Cochrane Central Register of Controlled Trials and Database of Systematic Reviews. Pooling was conducted by both fixed and random effects model.

\section{Results}

The initial search identified 543 reference articles; of these 24 relevant articles were selected and reviewed. Data was extracted from four studies $(n=136)$ which met the inclusion criteria. In the pooled analysis, the 90-day survival in the G-CSF group was $80.03 \%$ (95\% CI $=69.93-88.49$ ) compared to $40.92 \%$ (95\% CI $=29.76$ 52.58 ) in the Standard Medical Therapy (SMT) group. At 28 days, the Model for End-Stage Liver Disease (MELD) score lowered by 4.89 (95\% CI $=4.13-5.64)$ in the G-CSF group compared to $4.00(95 \% \mathrm{CI}=3.25-4.75)$ in the SMT group. Child-Turcotte-Pugh score declined by 2.26 (95\% CI = 1.90-2.63) in the G-CSF group after 28 days compared to 0.91 (95\% CI $=0.59-1.23$ ) in the SMT group. At 28 days, Maddrey Discriminant Function score lowered by 39.79 ( $95 \% \mathrm{CI}=34.22-45.36)$ in the $\mathrm{G}$-CSF group compared to $12.39(95 \% \mathrm{CI}=6.90-17.88)$ in the SMT group.

\section{Conclusions}

In patients with severe alcoholic hepatitis, G-CSF therapy resulted in significantly improved 90-day survival compared to SMT. It also demonstrated significant reduction in severity indices (Child-Turcotte-Pugh, MELD, and Maddrey discriminant function) after 28 days of treatment. There certainly is a need for further

Received 08/27/2020

Review began 09/09/2020 Review ended 09/12/2020 Published 09/15/2020

\section{(c) Copyright 2020}

Baig et al. This is an open access article distributed under the terms of the Creative Commons Attribution License CC-BY 4.0., which permits unrestricted use, distribution, and reproduction in any medium, provided the original author and source are credited.
Categories: Internal Medicine, Gastroenterology, Hematology

Keywords: alcoholic hepatitis, granulocyte colony-stimulating factor, systemic steroids

\section{Introduction}

Alcoholic hepatitis $(\mathrm{AH})$ has a very high mortality rate, approaching $40 \%$ within the first four weeks of clinical presentation [1]. Clinical diagnosis is made in the setting of worsening jaundice in a patient with chronic heavy alcohol use until at least six weeks prior to presentation, elevated liver enzymes, and exclusion of other liver diseases. However, whenever the clinical diagnosis is uncertain, liver biopsy may be needed for further diagnostic clarity [2]. Model of End-Stage Liver Disease (MELD) and Maddrey Discriminant Function (mDF) are commonly used in clinical practice to stratify the severity of an episode of AH and estimate prognosis. MELD scores of $>20$, mDF scores of $>32$, or the presence of hepatic encephalopathy indicate severe disease $[3,4]$.

Multiple therapeutic modalities have been investigated for the treatment of such patients. Corticosteroid therapy is the current first-line treatment for severe AH but has remained controversial due to modest reductions in 28-day mortality and no survival benefit beyond one month [5]. Moreover, due to the high incidence of systemic side effects and various absolute and relative contraindications, most treating physicians have to limit their use to only a fraction of these patients [6,7]. The Steroids or Pentoxifylline for Alcoholic Hepatitis (STOPAH) trial also showed no significant morbidity or mortality benefit with pentoxifylline, an alternative therapy for severe $\mathrm{AH}$ [8]. Anti-tumor necrosis factor agents, growth factors, 
and antioxidants were initially deemed promising but later found to be ineffective in further clinical studies as well $[1,9]$. Literature reveals encouraging results with salvage liver transplantation for patients with severe AH who remain non-responders $[10,11,12]$. However, stringent inclusion and exclusion criteria limits its utility to only 1 to $2 \%$ of cases $[10,13]$. Amidst all the confusion and unmet need for new therapeutic options, granulocyte colony-stimulating factor (G-CSF) has shown some promise to salvage the remaining patients with severe $\mathrm{AH}$, but the evidence remains limited at best.

G-CSF is an indigenous glycoprotein produced by macrophages, fibroblasts, and endothelial cells which promotes cell growth, differentiation, and function of neutrophils [14]. In human and animal trials, the administration of G-CSF to subjects with liver damage promoted liver repair by increasing the migration of bone marrow progenitors to the liver with clinical, biochemical, and histological improvement $[15,16]$.

There are currently several small reports suggesting benefit of G-CSF therapy in severe AH. The aim of the present meta-analysis was to systematically review the published literature and assess the efficacy of G-CSF therapy in improving survival and liver severity indices in patients with severe $\mathrm{AH}$.

\section{Materials And Methods Search methodology}

A literature search was conducted using the electronic database engines MEDLINE through PubMed, Ovid, Cochrane Library (Cochrane Central Register of Controlled Trials and Cochrane Database of Systematic Reviews), EMBASE, Cumulative Index for Nursing \& Allied Health Literature, ACP Journal Club, Database of Abstracts of Reviews of Effects (DARE), International Pharmaceutical Abstracts, OVID Healthstar, and Google Scholar from January 1974 to August 2020 to identify published articles and reports addressing the use of G-CSF in patients with AH. The combinations of keywords used were ("alcoholic hepatitis" or "alcoholic steatohepatitis") and ("Granulocyte colony-stimulating factor" or "G-CSF"). The reference list of all eligible studies was reviewed to identify additional studies. The retrieved studies were carefully examined to exclude potential duplicates or overlapping data. Titles and abstracts selected from the initial search were first scanned, and the full papers of potential eligible studies were reviewed.

\section{Study eligibility}

Published studies were eligible for inclusion if they reported the use of G-CSF for the management of severe alcoholic hepatitis. Articles were excluded if they were not written in English, no outcomes were reported, or they represented review articles or studies published as abstracts only. In studies using multiple modalities for the management of alcoholic hepatitis, data from the cohort of patients who received G-CSF were collected and analyzed. Two reviewers (MB, SP) independently performed study selection according to eligibility criteria. Disagreements were resolved by discussion or a third reviewer. The agreement between reviewers for the collected data gave a Cohen $\mathrm{K}$ value of 1.0.

\section{Data extraction and quality assessment}

The following data were independently abstracted onto a standardized form: study characteristics (primary author, time period of study, year of publication, and country of the population studied), study design, baseline characteristics of the study population (the numbers of patients enrolled, participant demographics, biochemical markers, and risk stratification scores when available), the intervention details and outcomes (change in biochemical markers, clinical severity indices, and survival). Risk of bias was rated for each study by two authors independently, using the Cochrane criteria for RCTs [17].

\section{Outcome definition}

The primary outcome of interest was 90-day survival of severe AH patients receiving G-CSF therapy compared to Standard Medical Therapy (SMT). Effect of G-CSF therapy on severity indices [Child-TurcottePugh (CTP), Model of End-stage Liver Disease (MELD) and Maddrey discriminant function (mDF)] was evaluated as a secondary outcome [18].

\section{Statistical analysis}

This meta-analysis was performed by calculating pooled proportions. First the individual study proportions were transformed into a quantity using Freeman-Tukey variant of the arcsine square root transformed proportion. The pooled proportion is calculated as the back-transform of the weighted mean of the transformed proportions, using inverse arcsine variance weights for the fixed effects model and DerSimonian-Laird weights for the random effects model [19]. Forest plots were drawn to show the point estimates in each study in relation to the summary pooled estimate. The width of the point estimates in the Forest plots indicates the assigned weight to that study. The heterogeneity among studies was tested using $\mathrm{I}^{2}$ statistic and Cochran Q test based upon inverse variance weights [20]. $\mathrm{I}^{2}$ of $0 \%$ to $39 \%$ was considered as nonsignificant heterogeneity, $40 \%$ to $75 \%$ as moderate heterogeneity, and $76 \%$ to $100 \%$ as considerable heterogeneity. If P-value is $>0.10$, it rejects the null hypothesis that the studies are heterogeneous. The effect of publication and selection bias on the summary estimates was tested by 


\section{Cureus}

Harbord-Egger bias indicator [21].

\section{Results}

The initial search identified 543 reference articles, of which 24 articles were selected and reviewed. Data were extracted from four studies that evaluated the efficacy of G-CSF in severe alcoholic hepatitis, which met the inclusion criterion [22,23,24,25]. A Preferred Reporting Items for Systematic Reviews and MetaAnalyses (PRISMA) flow diagram for details of the review process is shown in Figure 1 [26]. All included studies were randomized controlled trials and published in English as full-text articles. Pooled estimates were calculated by the fixed effect model for better accuracy based on the nature of individual study characteristics and heterogeneity. The P for Chi-squared heterogeneity for all the pooled accuracy estimates was >.10.
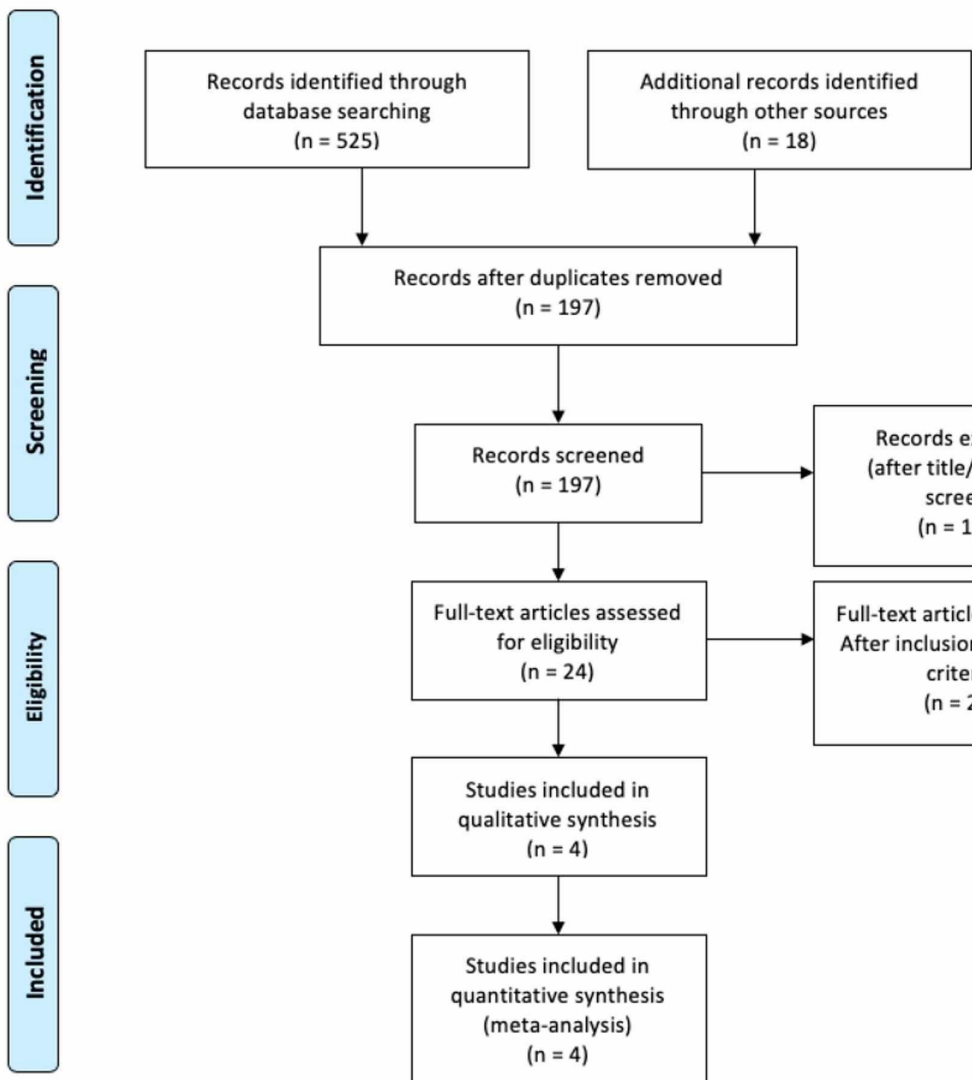

$(n=525)$

$$
(n=18)
$$
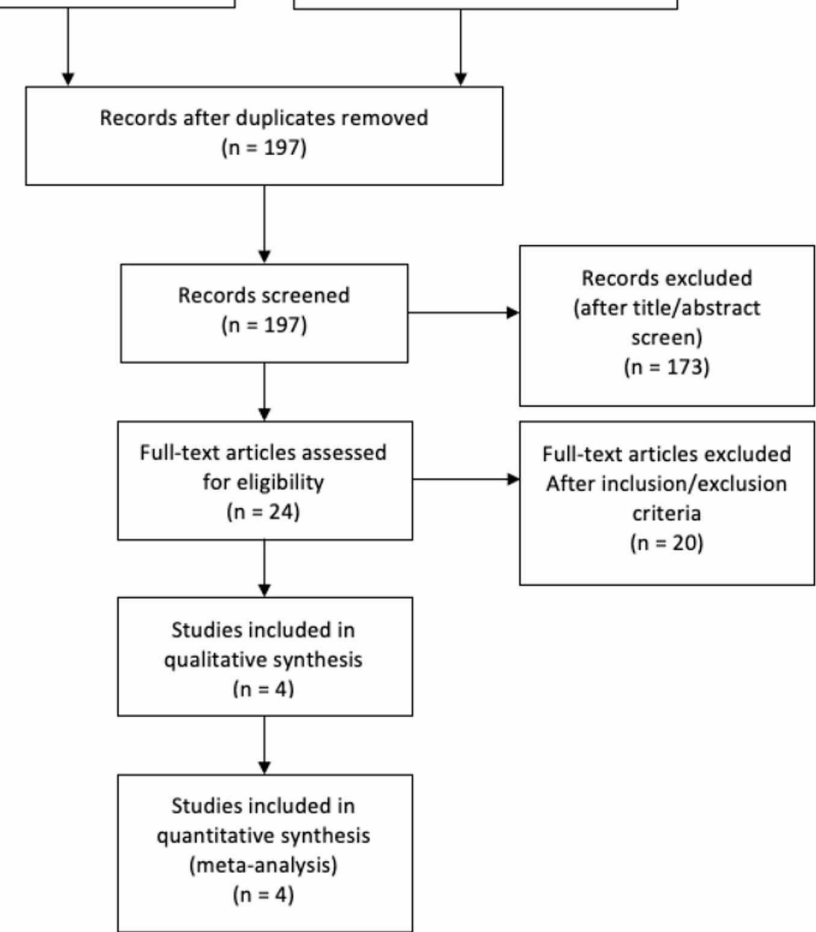

FIGURE 1: Preferred Reporting Items for Systematic Reviews and MetaAnalyses flow diagram detailing the review process.

The total number of patients included in this meta-analysis was 136. Mean age of the patients in the G-CSF group was 43.44 years compared to 45.32 years in the SMT group. Three studies used G-CSF dose of 10 $\mathrm{mcg} / \mathrm{kg}$ for five consecutive days $[23,24,25]$. One trial used $5 \mathrm{mcg} / \mathrm{kg}$ daily for five days followed by every third day until four weeks [22]. Table 1 shows the baseline characteristics of the studies. 


\section{Cureus}

\begin{tabular}{|c|c|c|c|c|c|c|}
\hline No. & Study & Year & Type & $\mathbf{N}$ & N-GCSF & N-Placebo \\
\hline 1 & Shasthry et al [22] & 2019 & $\mathrm{RCT}$ & 28 & 14 & 14 \\
\hline 2 & Singh et al [23] & 2014 & $\mathrm{RCT}$ & 46 & 23 & 23 \\
\hline 3 & Singh et al [24] & 2018 & RCT & 38 & 18 & 20 \\
\hline 4 & Spahr et al [25] & 2008 & RCT & 24 & 13 & 11 \\
\hline
\end{tabular}

TABLE 1: Basic characteristics of the included studies.

$\mathrm{N}=$ total number of patients in each study, N-GCSF = number of patients in each study receiving granulocyte colony-stimulating factor (GCSF), NPlacebo $=$ number of patients in each study receiving placebo with standard of care, RCT = randomized controlled trial.

\section{Primary outcome (90-day survival)}

In the pooled analysis, 90-day survival in the G-CSF group was 80.03\% (95\% CI $=69.93-88.49$ ) compared to $40.92 \%(95 \% \mathrm{CI}=29.76-52.58)$ in the SMT group with low heterogeneity $\left(\mathrm{I}^{2}=20.3 \%\right)$. Figures 2,3 are forest plots representing the pooled and individual 90-day survival rates with G-CSF therapy and SMT, respectively. Publication bias calculated using Harbord-Egger bias indicator gave a value of $-5.23(95 \% \mathrm{CI}=-$ 15.35-4.9, $\mathrm{P}=0.16$ ), indicating no publication bias.

\section{Proportion meta-analysis plot [fixed effects]}

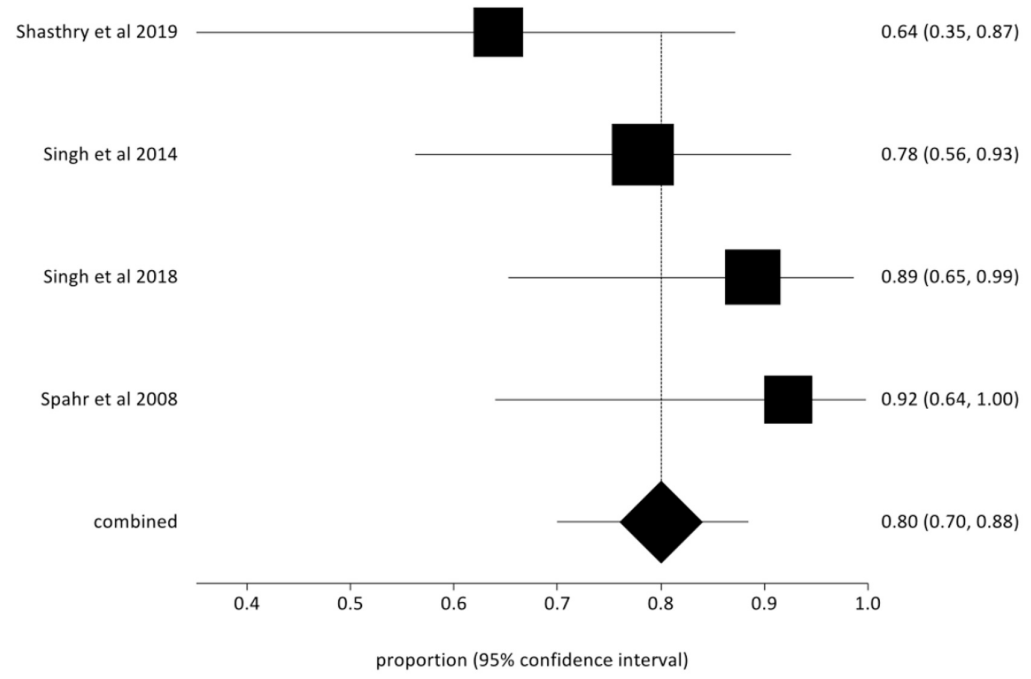

FIGURE 2: Forest plot-Individual study proportions and the pooled estimate of 90-day survival with granulocyte colony stimulating factor (G-CSF) therapy in severe alcoholic hepatitis (AH) (fixed effects). 


\section{Cureus}

Proportion meta-analysis plot [fixed effects]

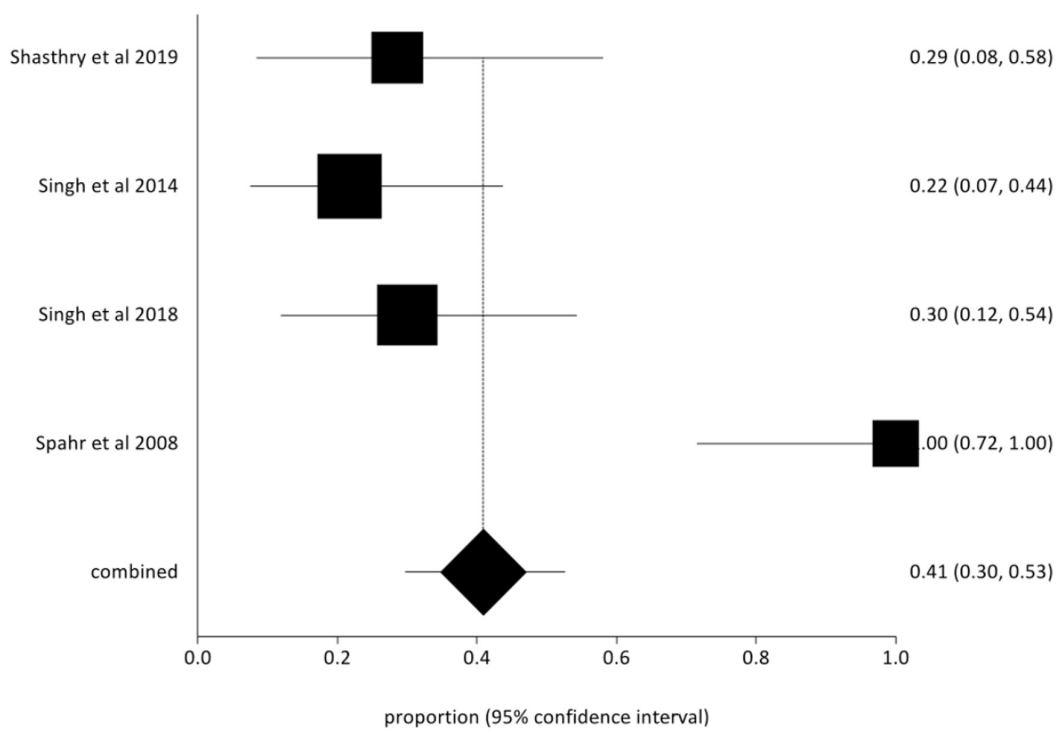

FIGURE 3: Forest plot-Individual study proportions and the pooled estimate of 90-day survival with placebo and standard of care therapy in severe alcoholic hepatitis (AH) (fixed effects).

\section{Secondary outcomes}

MELD Score

At 28 days, G-CSF therapy lowered the MELD score by 4.89 (95\% CI $=4.13-5.64)$ compared to a reduction of $4.00(95 \% \mathrm{CI}=3.25-4.75)$ in the SMT group. Moreover, at 90 days G-CSF therapy further reduced the MELD score by $11.94(95 \% \mathrm{CI}=11.04-12.84)$ compared to baseline.

Child-Turcotte-Pugh Score

At 28 days, G-CSF therapy lowered the CTP score by $2.26(95 \% \mathrm{CI}=1.90-2.63)$ which was significantly better than a reduction of 0.91 ( $95 \% \mathrm{CI}=0.59-1.23)$ in the SMT group. Moreover, at 90 days G-CSF therapy further lowered the CTP score by 3.21 ( $95 \%$ CI = 2.85-3.57) compared to baseline.

Maddrey Discriminant Function Score

At 28 days, G-CSF therapy lowered the $\mathrm{mDF}$ score by 39.79 (95\% CI $=34.22-45.36)$ which was significantly better than a reduction of $12.39(95 \% \mathrm{CI}=6.90-17.88)$ in the SMT group. Moreover, at 90 days G-CSF therapy further lowered the $\mathrm{mDF}$ score by $65.07(95 \% \mathrm{CI}=60.16-69.97)$ compared to baseline.

\section{Discussion}

Severe alcoholic hepatitis remains a commonly encountered clinical entity with high degree of morbidity and mortality and no satisfactory therapeutic option. Corticosteroid therapy remains the standard of medical care but literature supports only modest reductions in 28-day mortality with no long-term survival benefit [5]. High incidence of systemic side effects and risk of infectious complications likely contribute to lack of significant mortality reduction [6,7]. Studies on pentoxifylline, anti-TNF agents, growth factors, and antioxidants also failed to demonstrate any survival benefit in this high-risk patient population $[1,8,9]$.

Recombinant G-CSF, first approved in 1991 to reduce the incidence of neutropenic fever after myelosuppressive chemotherapy, causes proliferation and differentiation of bone marrow precursor cells into mature granulocytes [27]. Further studies have suggested its potential role in differentiation of bone marrow precursor cells into hepatocytes promoting liver regeneration $[15,16,25]$. The evidence regarding the latest is not universally conclusive, since some studies have been unable to reproduce these findings $[28,29]$. However, the effects of G-CSF in liver regeneration are not limited to the mobilization and differentiation of bone marrow-derived stem cells. G-CSF also exerts autocrine and paracrine effects in the liver, promoting and enhancing the oval cell reaction [15]. The synergistic contribution of the bone marrow- 
derived stem cells and the oval cells might be responsible for the liver function improvement observed in the cited studies. Spahr et al. first demonstrated the efficacy of G-CSF therapy in patients with alcoholic hepatitis, liver failure, and cirrhosis [25]. Chavez-Tapia et al. have also reported short-term mortality reduction and improvement in liver function with G-CSF therapy specifically in acute on chronic liver disease patients [30]. However, the evidence supporting G-CSF therapy to salvage patients with AH still remains limited at best.

Several case reports and small studies have investigated the use of G-CSF in severe AH. To the best of our knowledge, this is the first systematic review and meta-analysis to assess its role in improving survival and liver severity indices of patients with severe AH. Patients treated with G-CSF were noted to have significantly improved 90-day survival compared with standard medical therapy (80.03\% versus $40.92 \%$ ). This significant survival benefit is likely due to the role of G-CSF therapy in liver regeneration with reduced incidence of infectious complications compared to corticosteroids [22]. As the mobilization of hematopoietic stem cells occurs slowly over time, the survival benefit of G-CSF therapy is also long-term as seen in the prior literature and confirmed by our meta-analysis [16,23,25].

All included studies and their pooled analysis provided evidence of improved liver function demonstrated by significant reduction in CTP, MELD, and $\mathrm{mDF}$ scores at 28 days. The robust decline of mDF score by 39.79 in the G-CSF group compared to 12.39 in the SMT group is reassuring. All included studies reported continued improvement of liver function with G-CSF therapy even at 90 days. No significant adverse events were reported in any of the trials. Deaths reported among patients receiving G-CSF therapy were owing to progressive liver failure with no mortality reported directly as a complication of therapy [24]. As expected, there was an increase in neutrophil count with G-CSF therapy and only two patients required dose reduction due to significant leukocytosis (over $40,000 / \mathrm{cm}^{3}$ ) [23]. Singh et al. reported significant increase in spleen size with G-CSF therapy but no patient progressed to develop splenic rupture [24]. Minor bone pains were reported in two studies and only one patient reported by Shastry et al. required a reduction in the frequency and number of G-CSF doses to counter this side effect [22,25].

Our meta-analysis, however, has some important limitations. The number of trials included in this review is small and restricted to South Asian (India) and European populations, limiting the external validity of the results $[22,23,24,25]$. It is possible that the diagnosis of AH might have been incorrect in a small number of the patients due to absence of diagnostic confirmation with liver biopsy. The majority of the included trials did not report important information such as number of patients with underlying chronic liver disease, number of patients undergoing liver transplantation, and length of hospitalization, precluding the analysis of these important outcomes. Due to limited 90-day survival in the placebo arm, severity indices at 90 days were not reported in most of the studies and hence not included in our analysis.

\section{Conclusions}

In summary, G-CSF could be an additional rescue therapeutic option in patients with severe AH. It demonstrated a significant improvement of liver function and reduction in 90-day mortality with no significant adverse events. Based on the encouraging outcomes reported in the literature and our metaanalysis along with a long safety record of use in cancer patients, further randomized controlled trials evaluating the effectiveness of G-CSF in patients with alcoholic hepatitis are required.

\section{Additional Information}

\section{Disclosures}

Human subjects: All authors have confirmed that this study did not involve human participants or tissue. Animal subjects: All authors have confirmed that this study did not involve animal subjects or tissue. Conflicts of interest: In compliance with the ICMJE uniform disclosure form, all authors declare the following: Payment/services info: All authors have declared that no financial support was received from any organization for the submitted work. Financial relationships: All authors have declared that they have no financial relationships at present or within the previous three years with any organizations that might have an interest in the submitted work. Other relationships: All authors have declared that there are no other relationships or activities that could appear to have influenced the submitted work.

\section{Acknowledgements}

MB conceived of the study, performed the literature research and helped to draft the manuscript. SW performed the data extraction, and helped to draft the manuscript. SD provided general guidance and reviewed the final manuscript. SP participated in the study's design and data analysis and helped to draft the manuscript.

\section{References}

1. Singal AK, Kamath PS, Gores GJ, Shah VH: Alcoholic hepatitis: current challenges and future directions . Clin Gastroenterol Hepatol. 2014, 12:555-64. 10.1016/j.cgh.2013.06.013

2. Crabb DW, Bataller R, Chalasani NP, et al.: Standard definitions and common data elements for clinical 
trials in patients with alcoholic hepatitis: recommendation from the NIAAA alcoholic hepatitis consortia. Gastroenterology. 2016, 150:785-790. 10.1053/j.gastro.2016.02.042

3. Maddrey WC, Boitnott JK, Bedine MS, Weber Jr FL, Mezey E, White Jr RI: Corticosteroid therapy of alcoholic hepatitis. Gastroenterology. 1978, 75:193-199. 10.1016/0016-5085(78)90401-8

4. Dunn W, Jamil LH, Brown LS, et al.: MELD accurately predicts mortality in patients with alcoholic hepatitis . Hepatology. 2005, 41:353-358. 10.1002/hep.20503

5. Singh S, Murad MH, Chandar AK, et al.: Comparative effectiveness of pharmacological interventions for severe alcoholic hepatitis: a systematic review and network meta-analysis. Gastroenterology. 2015, 149:958970. 10.1053/j.gastro.2015.06.006

6. Singal AK, Salameh H, Singal A, et al.: Management practices of hepatitis C virus infected alcoholic hepatitis patients: a survey of physicians. World J Gastrointest Pharmacol Ther. 2013, 4:16-22. 10.4292/wjgpt.v4.i2.16

7. Raff E, Singal AK: Optimal management of alcoholic hepatitis. Minerva Gastroenterol Dietol. 2014, 60:2538.

8. Thursz MR, Richardson P, Allison M, et al.: Prednisolone or pentoxifylline for alcoholic hepatitis. N Engl J Med. 2015, 372:1619-1628. 10.1056/nejmoa1412278

9. Lucey MR, Mathurin P, Morgan TR: Alcoholic hepatitis. N Engl J Med. 2009, 360:2758-2769. 10.1056/nejmra0805786

10. Mathurin P, Moreno C, Samuel D, et al.: Early liver transplantation for severe alcoholic hepatitis. N Engl J Med. 2011, 365:1790-1800. 10.1056/NEJMoa1105703

11. Marot A, Dubois M, Trepo E, Moreno C, Deltenre P: Liver transplantation for alcoholic hepatitis: a systematic review with meta-analysis. PLoS One. 2018, 13:e0190823. 10.1371/journal.pone.0190823

12. Singal AK, Bashar H, Anand BS, Jampana SC, Singal V, Kuo YF: Outcomes after liver transplantation for alcoholic hepatitis are similar to alcoholic cirrhosis: exploratory analysis from the UNOS database. Hepatology. 2012, 55:1398-1405. 10.1002/hep.25544

13. Hasanin M, Dubay DA, McGuire BM, Schiano T, Singal AK: Liver transplantation for alcoholic hepatitis: a survey of liver transplant centers. Liver Transpl. 2015, 21:1449-1452. 10.1002/lt.24208

14. Houston AC, Stevens LA, Cour V: Pharmacokinetics of glycosylated recombinant human granulocyte colony-stimulating factor (lenograstim) in healthy male volunteers. Br J Clin Pharmacol. 1999, 47:279-284. 10.1046\%2Fj.1365-2125.1999.00888.x

15. Piscaglia AC, Shupe TD, Oh SH, Gasbarrini A, Petersen BE: Granulocyte-colony stimulating factor promotes liver repair and induces oval cell migration and proliferation in rats. Gastroenterology. 2007, 133:619-631. 10.1053/j.gastro.2007.05.018

16. Yannaki E, Athanasiou E, Xagorari A, et al.: G-CSF-primed hematopoietic stem cells or G-CSF per se accelerate recovery and improve survival after liver injury, predominantly by promoting endogenous repair programs. Exp Hematol. 2005, 33:108-119. 10.1016/j.exphem.2004.09.005

17. Cochrane Handbook for Systematic Reviews of Interventions . Higgins JPT, Green S (ed): John Wiley \& Sons, 2008. 10.1002/9780470712184

18. Rahimi E, Pan JJ: Prognostic models for alcoholic hepatitis. Biomark Res. 2015, 3:20. 10.1186\%2Fs40364015-0046-Z

19. DerSimonian R, Laird N: Meta-analysis in clinical trials. Control Clin Trials. 1986, 7:177-188. 10.1016/01972456(86)90046-2

20. Deeks JJ: Systematic reviews of evaluations of diagnostic and screening tests . BMJ. 2001, 323:157-162. 10.1136/bmj.323.7305.157

21. Harbord RM, Egger M, Sterne JA: A modified test for small-study effects in meta-analyses of controlled trials with binary endpoints. Stat Med. 2005, 25:3443-57. 10.1002/sim.2380

22. Shasthry SM, Sharma MK, Shasthry V, Pande A, Sarin SK: Efficacy of granulocyte colony-stimulating factor in the management of steroid-monresponsive severe alcoholic hepatitis: a double-blind randomized controlled trial. Hepatology. 2019, 70:802-811. 10.1002/hep.30516

23. Singh V, Sharma AK, Narasimhan RL, Bhalla A, Sharma N, Sharma R: Granulocyte colony-stimulating factor in severe alcoholic hepatitis: a randomized pilot study. Am J Gastroenterol. 2014, 109:1417-1423. 10.1038/ajg.2014.154

24. Singh V, Keisham A, Bhalla A, et al.: Efficacy of granulocyte colony-stimulating factor and N-acetylcysteine therapies in patients with severe alcoholic hepatitis. Clin Gastroenterol Hepatol. 2018, 16:1650-1656. 10.1016/j.cgh.2018.01.040

25. Spahr L, Lambert JF, Rubbia-Brandt L, Chalandon Y, Frossard JL, Giostra E, Hadengue A: Granulocytecolony stimulating factor induces proliferation of hepatic progenitors in alcoholic steatohepatitis: a randomized trial. Hepatology. 2008, 48:221-229. 10.1002/hep.22317

26. Moher D, Liberati A, Tetzlaff J, Altman DG; PRISMA Group: Preferred reporting items for systematic reviews and meta-analyses: the PRISMA statement. Ann Intern Med. 2009, 151:264-269. 10.7326/00034819-151-4-200908180-00135

27. Hollingshead LM, Goa KL: Recombinant granulocyte colony-stimulating factor (rG-CSF). A review of its pharmacological properties and prospective role in neutropenic conditions. Drugs. 1991, 42:300-330. 10.2165/00003495-199142020-00009

28. Masson S, Harrison DJ, Plevris JN, Newsome PN: Potential of hematopoietic stem cell therapy in hepatology: a critical review. Stem Cells. 2004, 22:897-907. 10.1634/stemcells.22-6-897

29. Thorgeirsson SS, Grisham JW: Hematopoietic cells as hepatocyte stem cells: a critical review of the evidence. Hepatology. 2006, 43:2-8. 10.1002/hep.21015

30. Chavez-Tapia NC, Mendiola-Pastrana I, Ornelas-Arroyo VI, et al.: Granulocyte-colony stimulating factor for acute-on-chronic liver failure: systematic review and meta-analysis. Ann Hepatol. 2015, 14:631-641. 10.1016/S1665-2681(19)30757-4 